\title{
EL USO DE JUEGOS PARA ENSEÑAR INGLÉS COMO SEGUNDA LENGUA
}

\section{ARTÍCULO DE REVISIÓN}

DIAS, Adailton Di Lauro ${ }^{1}$

DIAS, Adailton Di Lauro. El uso de juegos para enseñar inglés como segunda lengua. Revista Científica Multidisciplinar Núcleo do Conhecimento. Año 04, Ed. 07, Vol. 03, págs. 69-76. Julio de 2019. ISSN:2448-0959

\section{ABSTRACTO}

Evaluando las prácticas docentes actuales e incluso observando aspectos históricamente metodológicos, los estudiantes hoy en día se enfrentan a una dificultad extrema para desarrollar las habilidades cognitivas y adquirir conocimientos en el sistema educativo actual. Tal vez esto no se deba a la ineficacia de los métodos, sino a la dinámica de las relaciones entre el estudiante y el medio ambiente. Diferentes grupos de edad requieren diferentes enfoques, que han cambiado a la condición de obsolescencia; pierden su efectividad significativamente, a medida que pasa el tiempo. Por lo tanto, los juegos en términos de edad y sujetos pueden ser una manera eficiente de mostrar contenidos debido a su universalidad. El presente artículo tiene como objetivo explicar las razones por las que los juegos constituyen una herramienta importante en la educación e indicar algunas formas de aplicar estas herramientas en el proceso de enseñanza y aprendizaje del inglés como segunda lengua.

Palabras clave: Juego, habilidad, lenguaje, emociones, proceso de aprendizaje.

\footnotetext{
${ }^{1}$ Máster en Educación Científica en la Universidad Unigrendal. Especialista en Inglés por FlJ-Integrated Colleges of Jacarepagua, Río de Janeiro (2007). Licenciado en Literatura por la Universidad de Estado de Bahía, Brasil. Profesor de Inglés y Portugués en IFRR-Brasil.
} 


\section{INTRODUCCIÓN}

Teniendo en cuenta el progreso tecnológico a un ritmo constante y, aún más, observando que las metodologías deben acompañar estos cambios con el propósito de la mejora constante, este artículo busca relacionar el uso de juegos con la práctica de enseñar inglés como segundo idioma. El estudio no se limita a un modelo específico del juego, sino a cualquier forma lúdica de simular la realidad. Por lo tanto, el análisis detalla algunos tipos de juegos y sus aspectos históricos señalando la estrecha relación entre ellos, el contexto y el proceso de aprendizaje. La propuesta actual puede converger a una creación de prácticas, que utilizan juegos para hacer que el acto de aprendizaje sea agradable para los estudiantes; también puede ayudarles a utilizar el contenido propuesto de manera fluida y eficiente en su vida diaria.

La falta de situaciones reales que contribuyan a la práctica de nuevos idiomas por parte de los estudiantes constituye una de las principales dificultades para el proceso de enseñanza del inglés. En el entorno del aula, diferentes actividades pueden satisfacer parte de esta brecha si el maestro desarrolla interés estudiantil en el aprendizaje, concentrándose en las clases y actividades expositivas. Al considerar las muchas posibilidades en un entorno donde los estudiantes pueden simular situaciones extracurriculares y entrar en el contexto de estas situaciones, se puede obtener un mejor resultado. Las perspectivas de orden conceptual en este estudio consideran la literatura indicada, pero principalmente, considera la experiencia del autor en la praxis pedagógica, principalmente en la enseñanza del inglés como segunda lengua. Por lo tanto, la opinión del autor y la observación sustituyen, en este estudio, la investigación de campo requerida habitualmente.

Basado en investigaciones de juegos para niños, juegos y otros conceptos y temas relacionados, hay diferentes formas de aplicación de este estudio en el ámbito pedagógico. Para Dantas (1998) "el término lúdico se refiere al acto de jugar (de manera libre e individual) y jugar (con respecto a una conducta social que implica reglas)". Sin embargo, la relación específica y dinámica entre el método y los estudiantes hacen necesaria la profundización y la dirección de los juegos para cada 
asignatura. Según ANTUNES (2003), para que los juegos sean aplicables para la práctica de la enseñanza efectiva se requiere análisis e investigación, ya que el resultado se puede revertir si no hay compatibilidad entre ellos y el objeto de aprendizaje. La literatura utilizada guía parte de la construcción de conceptos sin definir la aplicabilidad de los juegos. Por lo tanto, el autor sugiere ese uso práctico y su posible convergencia metodológica.

\section{ALGUNAS CONSIDERACIONES SOBRE LOS PASOS PARA EL APRENDIZAJE}

Un punto importante a tener en cuenta es el contexto y las condiciones, que se aplican a los mecanismos de aprendizaje. Con respecto a los juegos, el público es muy heterogéneo, dada la variedad de juegos existentes y el rango ilimitado relacionado con las edades de los estudiantes. El juego es una actividad espontánea, realizada por una o más personas y, de manera diferente al arte y el trabajo, puede considerarse como un medio de estimulación física o mental, a veces ambos. La existencia de los juegos se remonta a tiempos prehistóricos y cubre todos los niveles de experiencia, géneros y edades. Entre las etapas del aprendizaje, es importante entender que los obstáculos a la supervivencia son la justificación de los diferentes procesos. Por lo tanto, estos procesos se producen en una escala de complejidad creciente. La creación de las operaciones necesarias para superar estos obstáculos, pasa por fases diferentes y bien definidas, desde la infancia hasta la adolescencia.

La primera etapa comienza al nacer y generalmente dura hasta el comienzo de la adquisición del idioma (hasta alrededor de dieciocho meses de edad). Durante este período, comienza la formación de habilidades motoras y la percepción sensorial. La segunda etapa ocurre aproximadamente entre dos y cuatro años de edad y se define por la formación del pensamiento simbólico. El uso de muñecas, coches y otros objetos de naturaleza simbólica que simulan la realidad, constituyen parte de ese escenario en particular. Entre cuatro y ocho años de edad aparece la tercera etapa, donde comienza a formarse el pensamiento intuitivo y los objetos alrededor se convierten en los puntos de referencia. 
Automáticamente, esta intuición conduce a la cuarta etapa, que es la etapa donde los seres humanos aprenden a organizar operaciones concretas para asociar cualquier objeto al que se haga referencia a su significado. La cuarta fase dura entre cuatro y ocho años. La última etapa se completa durante la adolescencia, donde la construcción de operaciones mentales es suficiente para el pensamiento analítico. Tomando estos pasos y sus distinciones, es posible entender que la idoneidad de los juegos para diferentes grupos de edad puede satisfacer a la mayoría de sus miembros.

\section{UTILIZAR LOS JUEGOS COMO APOYO PARA ENSEÑAR INGLES}

Para WITTGENSTEIN (2001), el concepto de "juego" no puede limitarse a una sola definición, sino como una multiplicidad de escenarios para establecer una relación de semejanza familiar entre ellos. La definición de multiplicidad de Wittgenstein encuentra apoyo también en CALLOIS (1957), que da algunas características necesarias para que una actividad se defina como un juego. Las condiciones son que la actividad debe ser divertida, limitada en tiempo y lugar, de resultados impredecibles y que no sea productiva. Además, la actividad debe tener reglas diferentes de la vida cotidiana y debe ser ficticia, acompañada de la conciencia de una realidad diferente. Por lo tanto, los juegos deben acercarse a lo real al imaginario y al aula, o el espacio alternativo elegido debe ser un entorno adecuado para estas prácticas.

El uso de la repetición en los niveles de idioma, al enseñar inglés, debe ocurrir como el proceso de enseñanza de la lengua materna, incluso para adultos principiantes. A excepción del aprendizaje de la gramática, la técnica de aprender inglés con el dedo índice apuntando a imágenes u objetos es muy eficiente. El maestro debe realizar la presentación del idioma claramente y en los juegos, usted debe evitar las actividades que conducen al aburrimiento o la distracción. Al principio, tareas como la traducción y la gramática tienen muy poco mérito real, ya que el lenguaje escrito es secundario en este punto. El objetivo principal es vincular el lenguaje al contexto y al entorno real, abstracto o ficticio de los juegos.

La elección de los juegos debe guiarse por la aplicabilidad al grupo de edad en cuestión, la viabilidad técnica y el estado de seguimiento por parte del profesor en 
todas las etapas de la actividad. Por lo tanto, los juegos educativos de construcción o instrucción se pueden utilizar ya que cumplen con los requisitos anteriores. De acuerdo con el propósito, cualquier tipo de juego se puede utilizar dependiendo de varias condiciones, pero es importante capturar el interés de toda la comunidad involucrada. Los siguientes son algunos tipos de juego definido que se pueden utilizar:

\subsection{JUEGOS INTERACTIVOS E INTERCAMBIADOS}

Estos juegos se utilizan para unirse al miembro de un grupo. Ayudan a los participantes a memorizar información específica y promueven un ambiente relajado. Los jugadores se distraen, gusanos, liberan tensiones y superaron las reservas personales. Deben ser utilizados en las primeras fases del desarrollo de un grupo, al comenzar una reunión, después de un descanso y cada vez que el grupo parece empezar a cansarse, aburrirse o no motivarse.

La música y la danza se recomiendan en los juegos activos para sintonizar el grupo en la actividad propuesta. La decisión ideal es utilizar juegos cortos con mucha acción y un alto gasto de energía.

\subsection{JUEGOS DE TOUCH Y CONFIANZA}

Estos juegos ayudan a los participantes a ver a sí mismos lidiando con la confianza y la confianza en sus vidas. Según la organización cultural y el nivel de apertura de las personas, el grupo puede ir gradualmente a otros ejercicios que implican tacto. Los juegos de tacto y confianza deben ser utilizados con mucho cuidado; el instructor debe estar atento al momento y a las reacciones del grupo, así como de los participantes, ofreciéndoles recursos para hacer frente bien a los fuertes procesos psicológicos internos.

\subsection{JUEGOS DE CREATIVIDAD Y REFLECCION}

Son juegos que estimulan la expresión imaginaria, la intuición y la creatividad. En estos juegos, los participantes pueden notarse a sí mismos y mostrar abiertamente a 
otros lo que han descubierto sobre sí mismos, sobre el tema estudiado y sobre el grupo. Los jugadores se ponen en contacto con su interior y el interior de otros jugadores, notando lo que es más relevante en todos los niveles. Este tipo de juego debe ser utilizado cuando los grupos están completamente integrados, trabajando juntos y utilizando todas las condiciones para profundizar en el tema estudiado hasta ese momento.

\subsection{JUEGOS DE GESTIÓN}

Estos juegos concentran la atención de los jugadores en la planificación, la gestión de recursos, la simulación de situaciones y el aprendizaje de técnicas específicas. Este tipo de juego debe ser utilizado después de que el grupo está bien integrado para que puedan llegar al objetivo propuesto. A veces es común parecer algunas dificultades en el aprendizaje de la materia por lo que es muy importante que todo el ciclo de aprendizaje vivo se está trabajando completamente.

\subsection{JUEGOS DE CIERRE}

Estos juegos ayudan a las personas a tener la oportunidad de establecer su posición relacionada con un tema, con el grupo y con sigo mismos, transfiriendo lo que han conseguido durante el proceso de aprendizaje a su vida cotidiana. Los juegos de síntesis y finalización son aquellos que ritualizan, evalúan y formalizan lo que se ha hecho durante el trabajo. Es muy importante dejar claro, a cada participante, que se está terminando un ciclo y que otro está empezando.

\subsection{JUEGOS DE HABILIDAD Y ESTRATEGIA}

Se puede utilizar en cualquier paso del proceso o situaciones. Es importante destacar que el deporte se incluye en casi todas las modalidades explicadas anteriormente, pero debe utilizarse para involucrar al grupo sobre el aspecto del aprendizaje, pero sin abandonar el aspecto competitivo, que es precisamente lo que atrae al público de la mayoría de los grupos de edad. El juego, realizado como una actividad de placer, se constituye como una herramienta a estudiar y aplicar a la enseñanza del inglés 
principalmente porque se sabe que nuestros cerebros aprenden a través de un proceso de repetición, ensayo y velocidad (VILLA \& SANTANDER, 2003). El ensayo, o experimentación, es el análisis de la situación. La repetición da la práctica que conduce a la velocidad, o eficiencia y eficacia. La habilidad de cada estudiante señalará qué nivel de juego se debe utilizar.

Entendiendo, por lo tanto, las diferentes definiciones de los juegos y la cuestión de los principios de aprendizaje de los seres humanos, se hace evidente la condición de pleno uso de los mismos en las prácticas de enseñanza. El interés del estudiante en el aprendizaje se vincula a su interés en el juego propuesto en ese momento. El entorno creado por el juego, teniendo en cuenta también que las emociones que influyen en los seres humanos también promueven la asimilación, pueden abastecer la falta de situaciones reales. Por lo tanto, cuanto mayor sea la emoción causada en los juegos, mayor será la posibilidad de establecer el contenido propuesto.

Las emociones positivas promueven más aprendizaje (SISTO \& MARTINELLI, 2006), por lo que el sentimiento de alegría supera la tristeza o el dolor. El profesor, por su parte, comienza a actuar como mediador entre lo real y la imaginación representada por el juego y su resultado. El aula cambia de un lugar de discurso pedagógico a un laboratorio para compartir experiencias, lleno de posibilidades.

\section{CONCLUSIÓN}

Este estudio intentó proporcionar una alternativa al proceso de enseñanza del inglés como segundo idioma, a través de juegos, buscando agregar valores al contexto educativo actual. Enseñar un idioma, fuera de su entorno natural, es un proceso que requiere habilidad y creatividad por parte del profesional involucrado. Entonces, desde el momento de elegir los juegos hasta el análisis de los resultados, el profesor debe tener cuidado de monitorear el desarrollo del reconocimiento de los estudiantes, con el fin de no perder el objetivo real. El objetivo es no sólo desarrollar estrategias, sino también hacer que funcionen correctamente. 
Ciertamente, el asunto no termina. El estudio constante y el intercambio de experiencias siguen siendo responsabilidad de las personas involucradas en el proceso de enseñanza y aprendizaje. El mayor o menor uso y difusión de los procedimientos en los que se utilicen las prácticas sugeridas determinará el éxito de las mismas. Considerando el espacio educativo como algo a mejorar y, según su dinamismo, no puede contener los conceptos estáticos y obsoletos.

\section{REFERENCIAS}

ABERASTURY, A. A criança e seus jogos. Porto Alegre: Artes Médicas, 1992.

AFFONSO, R. M. Ludodiagnóstico. São Paulo: Plêiade, 1995.

AGUIAR, J. S. Jogos para o ensino de conceitos: leitura e escrita na pré-escola. Campinas: Papirus, 1998.

ANTUNES, C. Jogos para a estimulação das múltiplas inteligências. $12^{\underline{a}}$ edição. Petrópolis, RJ: Vozes, 2003.

CALLOIS, Roger. Les Jeux et les hommes. Paris: Gallimard, 1957.

DANTAS, H. Brincar e Trabalhar. In: KISHIMOTO, T. M. (org). Brincar e suas teorias. São Paulo: Pioneira, 1998.

SISTO, F. F. \& MARTINELLI, S. de C. (orgs.) Afetividade e dificuldades de aprendizagem: uma abordagem psicoeducacional. São Paulo: Vetor, 2006.

VILA, Magda \& SANTANDER, Marli. Jogos cooperativos no processo de aprendizagem acelerada. São Paulo: Qualitymark, 2003

WITTGENSTEIN, Philosophical Investigations, Blackwell Publishing Ltd., MA: 2001.

Enviado: Junio de 2019. 
Aprovate: Julio, 2019. 